\title{
SIDIS cross sections: perturbative and non-perturbative aspects
}

\author{
Mariaelena Boglione* \\ University of Turin and INFN-Sezione Torino, Via Giuria 1, 10125 Torino, Italy \\ E-mail: boglioneeto.infn.it
}

I will address the study of Semi-Inclusive Deep Inelastic Scattering (SIDIS) cross sections over a wide range of transverse momenta, and in particular in the region of low to moderate $q_{T}$, where the interplay between perturbative and non-perturbative effects become more relevant. Some realistic SIDIS kinematic will be considered where the commonly used prescription of "matching" through the so-called Y-factor cannot be applied.

QCD Evolution 2016

May 30-June 03, 2016

National Institute for Subatomic Physics (Nikhef), Amsterdam

\footnotetext{
* Speaker.
} 


\section{Introduction}

Describing a hadronic process at high resolution scale $Q$, over the whole range of transverse momenta $q_{T}$, is a highly non-trivial task. Collinear perturbative QCD calculations allow us to compute its cross section at large $q_{T}$, where $q_{T}>Q$, but fail at low $q_{T}$.

Let's consider, for example, the $q_{T}$ differential cross section typical of a Drell Yan (DY) process. Although fixed order calculations correctly reproduce its large $q_{T}$ behaviour, they cannot describe DY data at small $q_{T}$ : in fact, at Born level the cross section is vanishing while at order $\alpha_{s}$ it is badly divergent. Low energy DY data, instead, show that low $q_{T}$ cross sections are not divergent. Its $M^{2}$ dependence is remarkably well described by a Gaussian distribution, due to a successful interplay among the $1 / M^{2}$ behaviour of the Born cross section, the DGLAP evolution in $Q^{2}$ and kinematics. Considering the same DY processes at different (but still low) energies, one easily realizes that data keep being consistent with a Gaussian behaviour, but require larger and larger Gaussian widths with increasing $\sqrt{s}$. This is shown in Fig. 1 where the $q_{T}$ distributions from the E288 experiment [1] at three different c.m. energies are reported: from left to right, $\sqrt{s}=19.4$ $\mathrm{GeV}, \sqrt{s}=23.8 \mathrm{GeV}$ and $\sqrt{s}=27.4 \mathrm{GeV}$. In each panel, different ranges in $M$ can be successfully reproduced by the same Gaussian width, while increasing values of $\sqrt{s}$ indicate the need of growing Gaussian widths: from left to right $\left\langle k_{T}^{2}\right\rangle=0.47 \mathrm{GeV}^{2} / c^{2},\left\langle k_{T}^{2}\right\rangle=0.57 \mathrm{GeV}^{2} / c^{2},\left\langle k_{T}^{2}\right\rangle=0.71$ $\mathrm{GeV}^{2} / c^{2}$ respectively. On the other hand, as soon as $q_{T}$ grows larger, DY cross sections do not follow a Gaussian distribution anymore, and present the expected pertubative tail, well described by collinear QCD computations. This is shown in Fig. 2, where DY data from the CDF Collaboration [2], corresponding to $\sqrt{s}=1.96 \mathrm{TeV}$, are compared to a typical Gaussian distribution.

Diverging contributions in the range of lower $q_{T}$ are due to large logarithms arising from the emission of soft and collinear gluons; they need to be resummed in order to obtain the physical cross section. Here $\alpha_{s}$ cannot be used as the effective expansion parameter of the perturbative series; instead, in this region, a perturbative expansion in terms of logarithms is performed, and this perturbative series is then resummed into the so-called Sudakov exponential form factor. This is
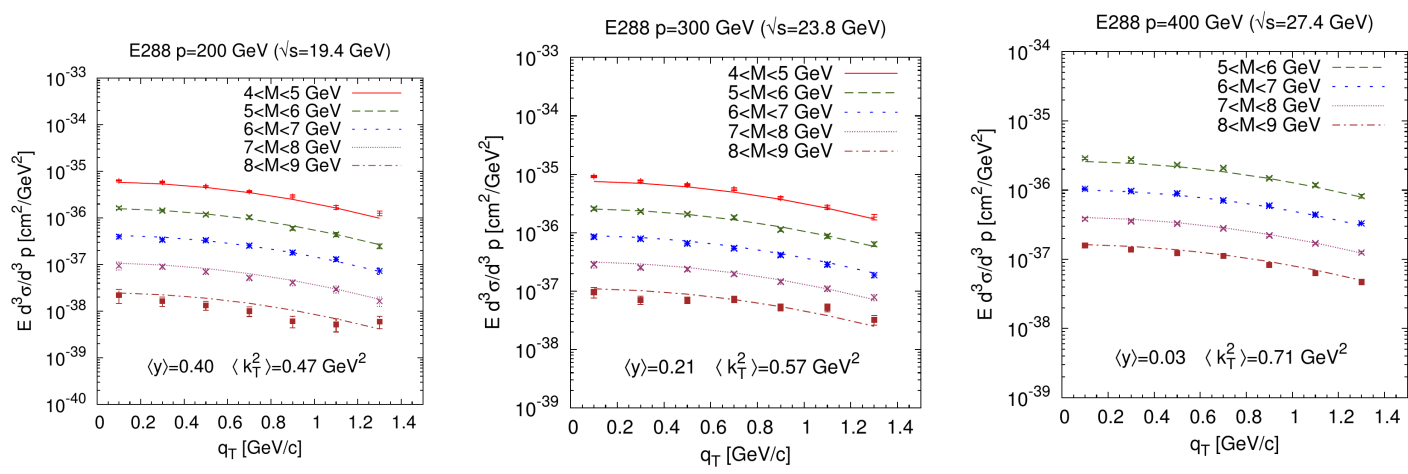

Figure 1: The $q_{T}$ distributions from the E288 experiment [1] at three different c.m. energies are reported: left panel $\sqrt{s}=19.4 \mathrm{GeV}$, central panel $\sqrt{s}=23.8 \mathrm{GeV}$, right panel $\sqrt{s}=27.4 \mathrm{GeV}$. At each fixed value of $\sqrt{s}$, i.e. in each panel, different ranges in $M$ can be successfully reproduced by the same Gaussian width, while increasing values of $\sqrt{s}$ indicate the need of growing Gaussian widths: left panel $\left\langle k_{T}^{2}\right\rangle=0.47 \mathrm{GeV}^{2} / c^{2}$, central panel $\left\langle k_{T}^{2}\right\rangle=0.57 \mathrm{GeV}^{2} / c^{2}$, right panel $\left\langle k_{T}^{2}\right\rangle=0.71 \mathrm{GeV}^{2} / c^{2}$. 


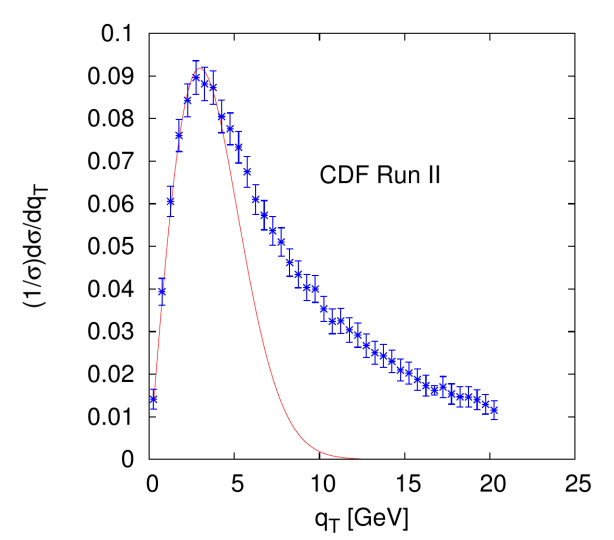

Figure 2: The $q_{T}$ distributions from the CDF Collaboration at Fermilab (Run II), at $\sqrt{s}=1.96 \mathrm{TeV}$. Data are from Ref. [2].

usually achieved by applying the Collins-Soper-Sterman (CSS) factorization scheme [3] or, equivalently, the improved Transverse Momentum Dependent (TMD) framework, developed more recently $[4,5]$. In fact, these two formalisms differ only at higher orders in $\alpha_{s}$, provided the auxiliary scales $\zeta_{F}$ and $\zeta_{D}$ are appropriately fixed so that $\zeta_{F}=\zeta_{D}=Q^{2}$. This equivalence was explicitly shown, for example, in Appendix B of Ref. [6].

Resummation is performed in the impact parameter space, $b_{T}$, the Fourier conjugate of the transverse momentum space, where momentum conservation can easily be applied. The cross section is then separated into two parts: the resummed term, W, which contains the whole essence of resummation itself, and the $\mathrm{Y}$ term, which is regular at small $q_{T}$ (i.e. less singular than $1 / q_{T}^{2}$ ). The CSS resummation scheme was originally developed for DY processes, but can be applied to SemiInclusive Deep Inelastic Scattering (SIDIS) reactions as well. For unpolarized SIDIS processes, $\ell N \rightarrow \ell h X$, the following CSS expression [7, 8] holds

$$
\frac{d \sigma^{\text {total }}}{d x d y d z d q_{T}^{2}}=\pi \sigma_{0}^{D I S} \int \frac{d^{2} \boldsymbol{b}_{T} e^{i \boldsymbol{q}_{T} \cdot \boldsymbol{b}_{T}}}{(2 \pi)^{2}} W^{S I D I S}\left(x, z, b_{T}, Q\right)+Y^{S I D I S}\left(x, z, q_{T}, Q\right),
$$

where $q_{T}$ is the virtual photon momentum in the frame where the incident nucleon $N$ and the produced hadron $h$ are head to head, and $\sigma_{0}^{D I S}$ is the LO elementary DIS cross section.

$$
\sigma_{0}^{D I S}=\frac{4 \pi \alpha_{\mathrm{em}}^{2}}{s x y^{2}}\left(1-y+\frac{y^{2}}{2}\right)
$$

with the usual DIS kinematical variables $x=Q^{2} /(2 P \cdot q), y=P \cdot q / P \cdot l$. Notice that, for SIDIS, we most commonly refer to the transverse momentum $\boldsymbol{P}_{T}$ of the final detected hadron, $h$, in the $\gamma^{*} N$ c.m. frame, rather than to the virtual photon momentum $\boldsymbol{q}_{T}$, in the $N h$ c.m. frame. They are simply related by the hadronic momentum fraction $z$ through the expression $\boldsymbol{P}_{T}=-z \boldsymbol{q}_{T}$, so that

$$
\frac{d \sigma}{d x d y d z d P_{T}^{2}}=\frac{d \sigma}{d x d y d z d q_{T}^{2}} \frac{1}{z^{2}}
$$

We will come back on this distinction below. At this stage, it is worth stressing that working in $b_{T}$ space makes phenomenological analyses more difficult, as we lose intuition and direct connection with "real world experience". In fact, experimental data are usually provided in momentum space. 

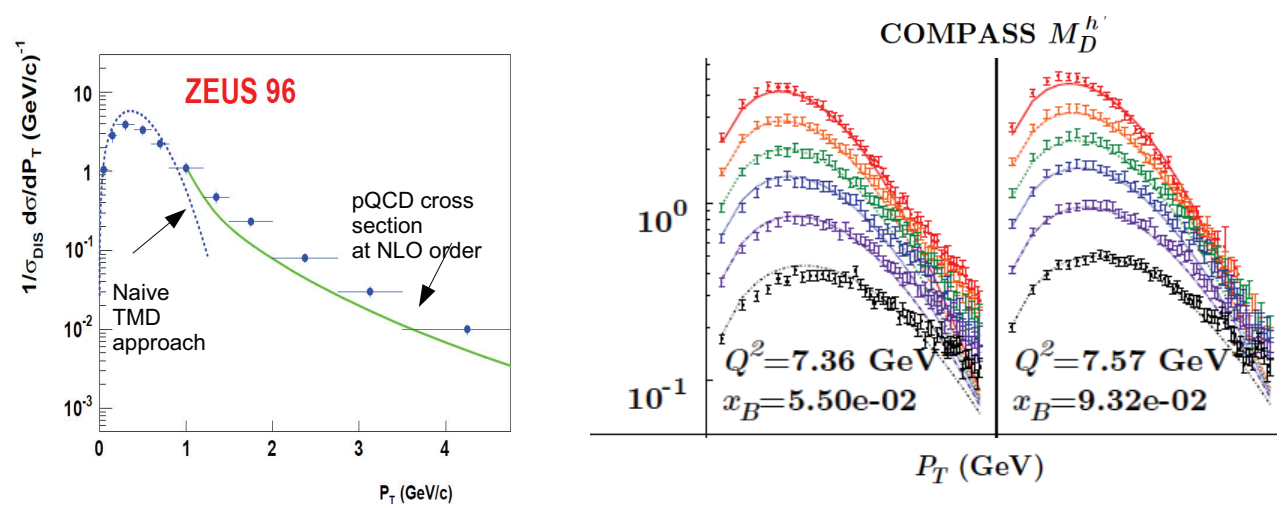

Figure 3: SIDIS cross sections show a Gaussian behaviour at very small $q_{T}$, where fixed order calculations diverge and cannot describe them correctly. However, they do not show a Gaussian tail at larger $q_{T}$, where perturbative QCD works well. The left panel is from Ref. [11], and shows the differential charged particle rates as a function of the transverse momentum of the produced hadron as measured by ZEUS detector at HERA [12], compared to the results presented in Ref. [11]. The right panel is from Ref. [9] and shows the COMPASS multiplicities distributions [13], in two bins of relatively large $Q^{2}$, compared to the results of the gaussian fit presented in Ref. [9].

A successful resummation scheme should take care of matching the fixed order hadronic cross section, computed in perturbative QCD at large $q_{T}$, with the so-called resummed cross section, valid at low $q_{T} \ll Q$, where large logarithms are properly treated. This matching should happen, roughly, at $q_{T} \sim Q$ where logarithms are small [3]. The regular Y-term, appropriately defined, should ensure a continuous and smooth matching of the cross section over the entire $q_{T}$ range.

Nevertheless, the perturbative resummed series does not converge at extremely low values of $q_{T}$, where we expect the transverse momentum to be "intrinsic" rather than generated by gluon radiation. Phenomenological analyses show that both Drell-Yan (DY) and SIDIS cross sections are consistent with a Gaussian behaviour at very small values of $q_{T}$. Recent analyses based on naive Gaussian models and extensive discussions on this subject can be found, for example, in Refs. $[9,10]$. However, neither DY nor SIDIS cross sections show a Gaussian tail at larger $q_{T}$ where, instead, perturbative QCD works well. This is illustrated, for SIDIS processes, in Fig. 3.

For the full description of the cross section, one should therefore be able to incorporate in the resummation scheme the non-perturbative behaviour as well. It is common to define $W^{N L L}$ the NLL resummed cross section which includes the non-perturbative Sudakov factor

$$
W^{N L L}=\pi \sigma_{0}^{D I S} \int_{0}^{\infty} \frac{d b_{T} b_{T}}{(2 \pi)} J_{0}\left(q_{T} b_{T}\right) W^{S I D I S}\left(x, z, b_{*}, Q\right) \exp \left[S_{N P}\left(x, z, b_{T}, Q\right)\right],
$$

with $W^{\operatorname{SIDIS}}\left(x, z, b_{*}, Q\right)$ calculated at NLL order. The non-pertubative part of the cross section is subject to phenomenological prescriptions and needs to be modeled. A commonly used parameterization is

$$
S_{N P}=\left(-\frac{g_{1}}{2}-\frac{g_{1 f}}{2 z^{2}}-g_{2} \ln \left(\frac{Q}{Q_{0}}\right)\right) b_{T}^{2} .
$$

Different values of the parameters $g_{1}, g_{1 f}$ and $g_{2}$, should, in principle, affect the hadronic cross section only in the range where $q_{T} \rightarrow 0$. Instead, it turns out that, for SIDIS processes like those 

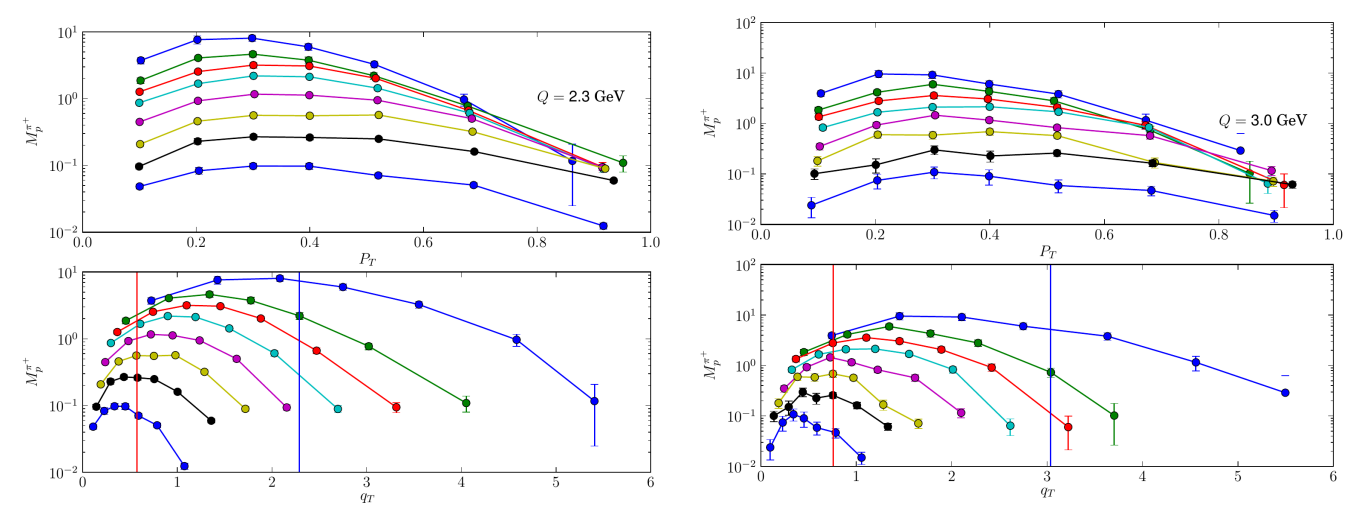

Figure 4: Two examples of data selection, according to $P_{T}$ (upper panels) and according to $q_{T}$ (lower panels), for $Q=2.3 \mathrm{GeV}$ (left panels) and $Q=3.0 \mathrm{GeV}$ (right panel).

measured by COMPASS [13] and HERMES [14] Collaborations, where $Q$ is only a few GeV's, the modeled non-perturbative contributions dominate over the entire range of measured $q_{T}$ 's. Therefore any resummation scheme would be inadequate in this case, and hardly applicable. This was shown and thoroughly discussed in Ref. [6].

Another controversial issue, which plays an important role in SIDIS phenomenological analyses, concerns the criteria used for data selection. The original CSS factorization scheme was derived for DY processes, where the relevant scales are $Q$ and $q_{T}$. Instead, as mentioned above, SIDIS differential cross sections are usually provided in terms of $P_{T}$, the transverse momentum of the measured final hadron $h$. Therefore, previous analyses of HERMES and COMPASS multiplicities were performed by selecting data, bin by bin, according to the value of $P_{T}$ as compared to their relative value of $Q$. However, it is important to point out that, as $P_{T}$ and $q_{T}$ are related by $\boldsymbol{P}_{T}=-z \boldsymbol{q}_{T}$, very different results are obtained by using $q_{T}$ rather than $P_{T}$, especially at low z. This effect is shown in Fig. 4, where the results obtained by cutting at $P_{T}<0.9$ are shown in the upper panels (for $Q=2.3 \mathrm{GeV}$ on the left and for $Q=3.0 \mathrm{GeV}$ on the right). The lower panels show the same data, plotted as a function of $q_{T}$ instead of $P_{T}$; here the vertical red lines correspond to $q_{T}=Q / 4$ while the blue vertical lines mark $q_{T}=Q$. From this plot one could conclude that the region corresponding to $q_{T} \ll Q$ should be roughly limited to the data points falling to the left of the red vertical lines, which, in turn, would lead to excluding most of the experimental information on the low $q_{T}$ behaviour of the SIDIS cross section. Moreover, in this case, one should expect the matching between the fixed order perturbative cross section and the resummed term to fall at (or around) the vertical blue line, where $q_{T} \sim Q$, i.e. just one or two GeVs above the "very small" $q_{T}$ region.

A pictorial representation of this situation, more in general, is given in Fig. 5 and can be summarized as follows. The TMD factorization scheme holds and can be applied when four different ranges of $q_{T}$ values can clearly be defined, and are neatly separated:

1. $q_{T} \sim \lambda_{Q C D}$, where the transverse momentum is expected to be "intrinsic";

2. $q_{T} \ll Q$, where TMD evolution is expected to be at work; 

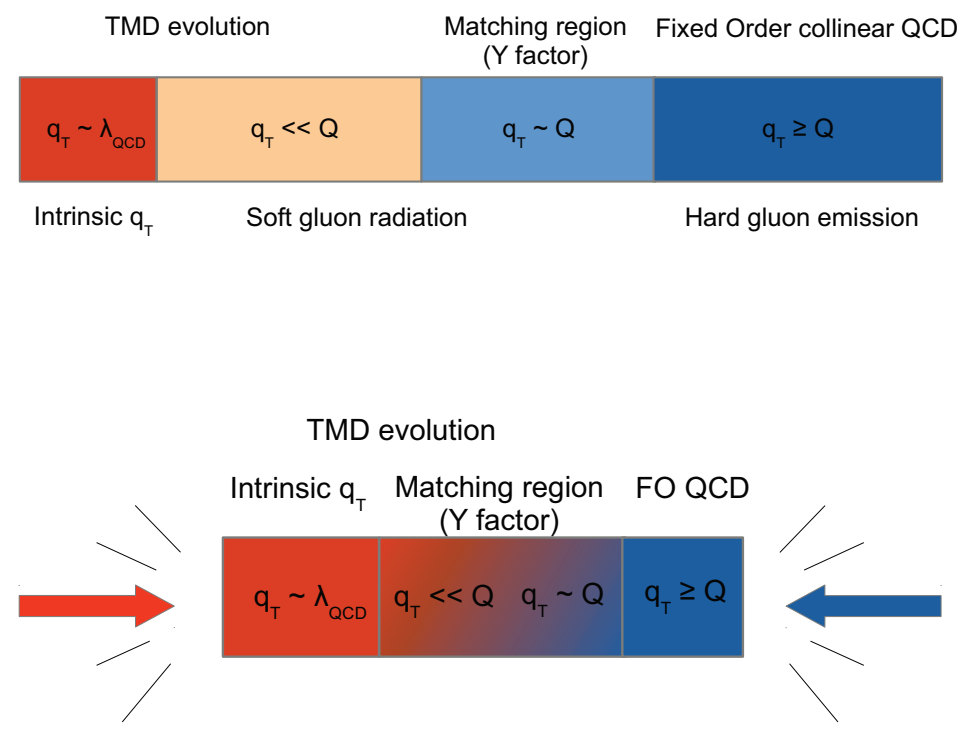

Figure 5: Different configurations of the $q_{T}$ ranges in a TMD factorization framework.

3. $q_{T} \sim Q$, where the matching between the fixed order perturbative cross section and the resummed term should take place;

4. $q_{T}>Q$, where the cross section can be computed perturbatively, at fixed order.

According to our more recent studies $[6,15,16]$, while in DY processes this is most usually the case, for SIDIS processes as measured at COMPASS and HERMES, where Q is limited to a few $\mathrm{GeVs}$, the $q_{T}$ range is extremely small, and all four regions are "compressed" and tend to overlap. Far from being neatly separated, they become very difficult to be identified: so much so that there is no room for resummation, nor space enough to perform a conventional matching. In this cases, non-perturbative effects completely dominate the cross section over the whole $q_{T}$ range.

Issues related to the identification of the current fragmentation region - the kinematical regime where a factorization picture with fragmentation functions is appropriate - has recently been addressed in Ref. [17], where general criteria for estimating the proximity to the current region at large $\mathrm{Q}$ are presented.

\section{Acknowledgments}

This talk is based on the work done in collaboration with J.O. Gonzalez-Hernandez, S. Melis and A Prokudin, whose endless efforts and incredible dedication are gratefully acknowledged.

\section{References}

[1] E288 Collaboration, A. S. Ito, et al., Measurement of the Continuum of Dimuons Produced in High-Energy Proton - Nucleus Collisions, Phys. Rev. D23 (1981) 604-633. 
[2] CDF Collaboration, T. Aaltonen et al., Transverse momentum cross section of $e^{+} e^{-}$pairs in the Z-boson region from $p \bar{p}$ collisions at $\sqrt{s}=1.96$ TeV, Phys. Rev. D86 (2012) 052010 [arXiv:1207.7138].

[3] J. C. Collins, D. E. Soper, and G. F. Sterman, Transverse Momentum Distribution in Drell-Yan Pair and $W$ and Z Boson Production, Nucl.Phys. B250 (1985) 199.

[4] J. Collins Foundations of perturbative QCD (2011) Cambridge monographs on particle physics, nuclear physics and cosmology, n. 32, Cambridge University Press, Cambridge.

[5] S. M. Aybat and T. C. Rogers, TMD Parton Distribution and Fragmentation Functions with QCD Evolution, Phys.Rev. D83 (2011) 114042, [arXiv: 1101.5057 ].

[6] M. Boglione, J. O. G. Hernandez, S. Melis, and A. Prokudin, A study on the interplay between perturbative QCD and CSS/TMD formalism in SIDIS processes, JHEP 02 (2015) 095, [arXiv:1412.1383].

[7] P. M. Nadolsky, D. R. Stump, and C. P. Yuan, Semiinclusive hadron production at HERA: The Effect of QCD gluon resummation, Phys. Rev. D61 (2000) 014003, [hep-ph / 9906280 ]. [Erratum: Phys. Rev.D64,059903(2001)].

[8] Y. Koike, J. Nagashima, and W. Vogelsang, Resummation for polarized semi-inclusive deep-inelastic scattering at small transverse momentum, Nucl.Phys. B744 (2006) 59-79, [hep-ph / 0602188 ].

[9] M. Anselmino, M. Boglione, J. Gonzalez H., S. Melis, and A. Prokudin, Unpolarised Transverse Momentum Dependent Distribution and Fragmentation Functions from SIDIS Multiplicities, JHEP 1404 (2014) 005, [arXiv:1312.6261].

[10] A. Signori, A. Bacchetta, M. Radici, and G. Schnell, Investigations into the flavor dependence of partonic transverse momentum, JHEP 11 (2013) 194, [arXiv: 1309.3507 ].

[11] M. Anselmino, M. Boglione, A. Prokudin, and C. Turk, Semi-Inclusive Deep Inelastic Scattering processes from small to large P(T), Eur. Phys. J. A31 (2007) 373-381, [hep-ph/ 0606286 ].

[12] ZEUS Collaboration, M. Derrick et al., Inclusive charged particle distributions in deep inelastic scattering events at HERA, Z. Phys. C70 (1996) 1-16, [hep-ex/9511010].

[13] COMPASS Collaboration, C. Adolph et al., Hadron Transverse Momentum Distributions in Muon Deep Inelastic Scattering at 160 GeV/c, Eur. Phys. J. C73 (2013), no. 8 2531, [arXiv:1305.7317]. [Erratum: Eur. Phys. J.C75,no.2,94(2015)].

[14] HERMES Collaboration, A. Airapetian et al., Multiplicities of charged pions and kaons from semi-inclusive deep-inelastic scattering by the proton and the deuteron, Phys. Rev. D87 (2013) 074029, [arXiv:1212.5407].

[15] S. Melis, M. Boglione, J. Gonzalez Hernandez, and A. Prokudin, Theoretical Uncertainties and Dependence on the $C_{1}, C_{2}, C_{3}$ Parameters in the CSS Formalism in Drell-Yan and SIDIS, PoS QCDEV2015 (2015) 038.

[16] M. Boglione, J. O. Gonzalez-Hernandez, S. Melis, and A. Prokudin, Challenges in the Extraction of TMDs from SIDIS Data: Perturbative vs Non-Perturbative Aspects, PoS DIS2015 (2015) 196.

[17] M. Boglione, J. Collins, L. Gamberg, J.O. Gonzalez-Hernandez, T.C. Rogers, N. Sato, Kinematics of Current Region Fragmentation in Semi-Inclusive Deeply Inelastic Scattering,

[arXiv:1611.10329]. 